\title{
PREVALÊNCIA DOS FATORES DE RISCO PARA O INFARTO AGUDO DO MIOCÁRDIO NA PARAÍBA
}

\author{
Maria Juliana Ferreira dos Santos ${ }^{1}$ \\ Milena Karen Tavares Nogueira ${ }^{2}$ \\ Elisangela Vilar de Assis ${ }^{3}$ \\ Juliane Carla Medeiros de Sousa ${ }^{4}$ \\ Marta Ligia Vieira Melo ${ }^{5}$
}

RESUMO: OBJETIVO: Identificar a prevalência dos fatores de risco para o infarto agudo do miocárdio no estado da Paraíba. MÉTODO: Trata-se de um estudo ecológico de séries temporais. Os dados foram coletados no Sistema de Informação de Notificação de Agravos de Notificação (SINAN), no período estabelecido entre abr/2009 - abr/2013. RESULTADOS: Conforme os resultados observa-se que a prevalência de pacientes com infarto agudo do miocárdio e hipertensão é maior entre os homens com idade de 60 a 64 anos e entre as mulheres, de 55 a 59 anos. Com relação aos fatores de risco para as doenças cardiovasculares destaca-se que as mulheres apresentaram prevalências maiores do que os homens em quase todos os fatores avaliados: sedentarismo, sobrepeso, tabagismo e doença renal. CONCLUSÃO: Portanto, observa-se que há uma diferença na prevalência entre os fatores de risco para o infarto agudo do miocárdio conforme o sexo, mostrando que medidas de prevenção e promoção da saúde devem ser instituídas com particularidades para cada sexo.

PALAVRAS CHAVE: Comportamento de risco a saúde. Doenças cardiovasculares. Isquemia.

\footnotetext{
${ }^{1}$ Acadêmica do $7^{\circ}$ período do Curso Bacharelado em Fisioterapia da Faculdade Santa Maria, Cajazeiras, PB. E-mail: mariajuliana.mjf@gmail.com.

2 Acadêmica do $7^{\circ}$ período do Curso Bacharelado em Fisioterapia da Faculdade Santa Maria, Cajazeiras, PB. E-mail: milenakaren1998@hotmail.com.

${ }^{3}$ Doutora em Ciências pela Faculdade de Medicina do ABC, Santo André, SP. Docente Universidade Federal de Campina Grande - UFCG/Campus Cajazeiras, Cajazeiras, PB. E-mail: ely.vilar@hotmail.com.

${ }^{4}$ Mestre em Saúde Coletiva pela Universidade de Santos, Santos, SP. Docente da Faculdade Santa Maria, Cajazeiras, PB. E-mail: julianecarlam@gmail.com.

${ }^{5}$ Mestre em Saúde Coletiva pela Universidade de Santos - UNISANTOS, Santos, SP. Docente da Faculdade Santa Maria, Cajazeiras, PB. E-mail: martaligiafisio@hotmail.com.
} 


\section{INTRODUÇÃO}

As doenças cardiovasculares (DCV), entre elas o infarto agudo do miocárdio (IAM), é responsável por cerca de $32 \%$ dos óbitos no Brasil, sendo uma das principais causas de morte e o maior problema de saúde pública no mundo. Além de que, constituem a terceira maior causa de internações no Brasil (TUAN; VENÂNCIO; NASCIMENTO, 2016). A perspectiva para o ano de 2020 é de que a doença arterial coronariana estará relacionada por cerca de $40 \%$ das mortes, sendo o IAM a principal causa (TEIXEIRA; SANCHES; VIVAS, 2017).

No estudo de Silveira et al. (2018), no qual analisaram os fatores de risco cardiovascular em pacientes com doença arterial coronariana no norte do Brasil, é possível observar que o perfil socioeconômico e educacional dos indivíduos esteja associado aos episódios de IAM, isto pode ser explicado pelo fato dos indivíduos com menos escolaridade possuírem menor conhecimento acerca dos métodos preventivos. Neste sentido, a população mais pobre é a mais vulnerável para as DCV. A hipertensão arterial sistêmica (HAS) é o fator de risco modificável mais prevalente e importante para desenvolver IAM, em conjunto com as dislipidemias. Ressalta-se ainda que, o sedentarismo, tabagismo, obesidade e por consequente, índice de massa corporal (IMC) elevado também se apresentam como importantes fatores de risco para o IAM.

Além de que, o risco de aparecimento das DCV aumenta em função da variação das concentrações dos poluentes atmosféricos, como $\mathrm{CO}_{2}$ e $\mathrm{SO}_{2}$ (TUAN; VENÂNCIO; NASCIMENTO 2016). Dentre os fatores de risco não modificáveis, a idade, a histórico familiar e o sexo são os principais aliados para o aparecimento da doença (TEIXEIRA; SANCHES; VIVAS, 2017).

Considerando-se a crescente porcentagem da população com DCV associado principalmente ao IAM, e tendo em vista a projeção futura deste cenário, torna-se evidente a importância de estudar os fatores de risco para esta doença. A investigação desses fatores de risco contribui para a aquisição de uma assistência apropriada e eficaz, favorável para a redução dos índices de morbidade e 
mortalidade da população. Sendo assim, o presente estudo tem como objetivo principal identificar a prevalência dos fatores de risco para o infarto agudo do miocárdio no estado da Paraíba.

\section{MÉTODO}

Trata-se de um estudo ecológico de séries temporais. Os dados foram coletados no Sistema de Informação de Notificação de Agravos de Notificação (SINAN), no período estabelecido entre abr/2009 - abr/2013 referentes ao estado da Paraíba por local de internação. O público foi paciente com diagnóstico de Infarto Agudo do Miocárdio (IAM) e expostos os fatores de risco.

Foram estabelecidos como fatores de risco: sedentarismo, sobrepeso, tabagismo, doença renal, idade e a presença de outras doenças coronarianas. Essas variáveis foram avaliadas conforme o sexo.

A análise dos dados foi realizada pelo programa SPSS versão 21, apresentando os resultados de forma descritiva por meio das frequências absolutas e relativas.

\section{RESULTADOS}

Conforme os resultados observa-se que a prevalência de pacientes com infarto agudo do miocárdio e hipertensão é maior entre os homens com idade de 60 a 64 anos e entre as mulheres, de 55 a 59 anos (Tabela 1). 
Tabela 1 - Prevalência de pacientes com infarto agudo do miocárdio e hipertensão arterial por faixa etária e sexo. Paraíba, Brasil - 2009 a 2013.

\begin{tabular}{ccccccc}
\hline \multirow{2}{*}{ Faixa Etária } & \multicolumn{2}{c}{ Masculino } & \multicolumn{2}{c}{ Feminino } & \multicolumn{2}{c}{ Total } \\
\cline { 2 - 7 } & $\mathbf{N}$ & $\%$ & $\mathbf{n}$ & $\%$ & $\mathbf{N}$ & $\%$ \\
\hline Até $\mathbf{1 4}$ & 3 & 0,3 & 8 & 0,6 & 11 & 0,5 \\
$\mathbf{1 5}$ a $\mathbf{1 9}$ & 6 & 0,7 & 3 & 0,2 & 9 & 0,4 \\
$\mathbf{2 0}$ a $\mathbf{2 4}$ & 7 & 0,8 & 9 & 0,7 & 16 & 0,7 \\
$\mathbf{2 5}$ a $\mathbf{2 9}$ & 13 & 1,5 & 20 & 1,5 & 33 & 1,5 \\
$\mathbf{3 0}$ a $\mathbf{3 4}$ & 20 & 2,3 & 42 & 3,2 & 62 & 2,8 \\
$\mathbf{3 5}$ a 39 & 25 & 2,9 & 72 & 5,4 & 97 & 4,4 \\
$\mathbf{4 0}$ a 44 & 61 & 7,0 & 106 & 8,0 & 167 & 7,6 \\
$\mathbf{4 5}$ a 49 & 83 & 9,5 & 161 & 12,2 & 244 & 11,1 \\
$\mathbf{5 0}$ a 54 & 87 & 10,0 & 172 & 13,0 & 259 & 11,8 \\
$\mathbf{5 5}$ a 59 & 107 & 12,3 & 175 & $\mathbf{1 3 , 2}$ & 282 & 12,8 \\
$\mathbf{6 0}$ a $\mathbf{6 4}$ & 118 & $\mathbf{1 3 , 5}$ & 156 & 11,8 & 274 & 12,5 \\
$\mathbf{6 5}$ a $\mathbf{6 9}$ & 113 & 13,0 & 125 & 9,4 & 238 & 10,8 \\
$\mathbf{7 0}$ a $\mathbf{7 4}$ & 88 & 10,1 & 96 & 7,3 & 184 & 8,4 \\
$\mathbf{7 5}$ a $\mathbf{7 9}$ & 54 & 6,2 & 62 & 4,7 & 116 & 5,3 \\
$\mathbf{8 0}$ e + & 86 & 9,9 & 117 & 8,8 & 203 & 9,2 \\
\hline Total & $\mathbf{8 7 1}$ & $\mathbf{1 0 0}$ & $\mathbf{1 3 2 4}$ & $\mathbf{1 0 0}$ & $\mathbf{2 1 9 5}$ & $\mathbf{1 0 0}$ \\
\hline
\end{tabular}

Fonte: Ministério da Saúde - Sistema de Cadastramento e Acompanhamento de Hipertensos e Diabéticos - 2019.

Com relação aos fatores de risco para as doenças cardiovasculares destacase que as mulheres apresentaram prevalências maiores do que os homens em quase todos os fatores avaliados (Tabela 2). 
Tabela 2 - Prevalência de pacientes com infarto agudo do miocárdio e hipertensão arterial por fator de risco e sexo. Paraíba, Brasil - 2009 a 2013.

\begin{tabular}{|c|c|c|c|c|c|c|}
\hline \multirow{2}{*}{ Fatores de Risco } & \multicolumn{4}{|c|}{ Sexo } & \multirow{2}{*}{\multicolumn{2}{|c|}{ Total }} \\
\hline & \multicolumn{2}{|c|}{ Masculino } & \multicolumn{2}{|c|}{ Feminino } & & \\
\hline Sedentarismo & $n$ & $\%$ & $\mathbf{N}$ & $\%$ & $\mathrm{n}$ & $\%$ \\
\hline Sim & 414 & 47,5 & 657 & 49,6 & 1.071 & 48,8 \\
\hline Não & 457 & 52,5 & 667 & 50,4 & 1.124 & 51,2 \\
\hline Total & 871 & 100 & 1324 & 100 & 2.195 & 100 \\
\hline Tabagismo & $\mathbf{n}$ & $\%$ & $\mathbf{N}$ & $\%$ & $\mathbf{N}$ & $\%$ \\
\hline $\operatorname{Sim}$ & 290 & 33,2 & 513 & 38,7 & 803 & 36,6 \\
\hline Não & 581 & 66,8 & 811 & 61,3 & 1392 & 63,4 \\
\hline Total & 871 & 100 & 1324 & 100 & 2195 & 100 \\
\hline Sobrepeso & $\mathbf{n}$ & $\%$ & $\mathbf{N}$ & $\%$ & $\mathbf{N}$ & $\%$ \\
\hline $\operatorname{Sim}$ & 292 & 33,5 & 561 & 42,4 & 853 & 38,9 \\
\hline Não & 579 & 66,5 & 763 & 57,6 & 1342 & 61,1 \\
\hline Total & 871 & 100 & 1324 & 100 & 2195 & 100 \\
\hline $\begin{array}{l}\text { Outras } \\
\text { coronarianas }\end{array}$ & $\mathbf{n}$ & $\%$ & $\mathbf{N}$ & $\%$ & $\mathbf{N}$ & $\%$ \\
\hline Sim & 251 & 28,8 & 336 & 25,4 & 587 & 26,7 \\
\hline Não & 620 & 71,2 & 988 & 74,6 & 1608 & 73,3 \\
\hline Doença renal & $\mathbf{n}$ & $\%$ & $\mathbf{N}$ & $\%$ & $\mathbf{N}$ & $\%$ \\
\hline Sim & 90 & 10,3 & 182 & 13,7 & 272 & 12,4 \\
\hline Não & 781 & 89,7 & 1142 & 86,3 & 1923 & 87,6 \\
\hline Total & 871 & 100 & 1324 & 100 & 2195 & 100 \\
\hline
\end{tabular}

Fonte: Ministério da Saúde - Sistema de Cadastramento e Acompanhamento de Hipertensos e Diabéticos - 2019.

\section{DISCUSSÃO}

No presente estudo foram analisados grupos no período de 2009 a 2013, que sofreram IAM e que apresentaram pelo menos um dos principais fatores de risco propícios para seu desenvolvimento. As pesquisas foram baseadas nos fatores: sexo, idade, sedentarismo, tabagismo, pessoas com sobrepeso, portadores de doença renal e portadores de outras doenças coronarianas.

Os achados deste estudo apresentam prevalência do infarto agudo do miocárdio em mulheres, esse fato pode ser atribuído à suscetibilidade de aumento do Índice de Massa Corporal, considerado comum para esse grupo. De acordo com 
Siqueira et al. (2015) a prevalência de obesidade abdominal é de 49,7\% e aumenta com a idade para ambos os sexos, nesse estudo foi observado que em indivíduos acima de 40 anos a prevalência em mulheres foi mais que o dobro da verificada em homens.

A pesquisa com base na faixa etária mostra que as mulheres estão suscetíveis a sofrerem de forma precoce um IAM quando comparado aos homens. Os dados analisados apresentam uma diferença de cinco anos entre os sexos, sendo mulheres com idade entre 55 e 59 anos enquanto os homens com idade de 60 a 64 anos.

De acordo com os resultados os números atribuídos à prevalência do sedentarismo às mulheres sedentárias foram maiores do que a dos homens sedentários, tornando-se um fator de risco importante para a ocorrência de IAM nesse grupo. Segundo Carvalho et al. (2018) os benefícios da atividade física na redução de risco de doença coronariana já estão bem consolidados na literatura. $\mathrm{O}$ sedentarismo é fator de risco independente e contribui para o desenvolvimento de outros fatores, pois está diretamente relacionado a níveis baixos de colesterol HDL, aumento de peso, aumento da pressão sanguínea e também de alguns fatores da coagulação.

Nesse mesmo comportamento, destaca-se que a prevalência do consumo do tabaco também foi maior entre as mulheres. De acordo com Luciano et al. (2007) a associação entre o tabagismo e maior ocorrência de doença coronariana aterosclerótica advém de múltiplos efeitos deletérios causados pelo cigarro, seu consumo afeta diversos mecanismos que dificultam a circulação sanguínea, se tornando um fator relevante.

Em apenas um dos fatores de risco analisados o sexo masculino se destaca, nos indivíduos que apresentam outras doenças coronarianas e desenvolveram IAM os homens apresentaram uma prevalência maior do que o sexo feminino. Durante o período de pesquisa do presente estudo esse fator de risco não foi o mais predominante, no entanto, tal possibilidade não pode ser descartada, visto o aumento da quantidade de casos IAM ao longo dos anos. 


\section{CONCLUSÃO}

Portanto, observa-se que há uma diferença na prevalência entre os fatores de risco para o infarto agudo do miocárdio conforme o sexo, mostrando que medidas de prevenção e promoção da saúde devem ser instituídas com particularidades para cada sexo.

\section{REFERÊNCIAS BIBLIOGRÁFICAS}

CARVALHO, A.T.G. et al. Correlação entre Atividade Física e Variáveis Clínicas de Pacientes com Infarto Agudo do Miocárdio. Int. J. Cardiovasc. Sci., v.31, n.1, p. 22-25, 2018.

LUCIANO, K.S. et al. Paradoxo dos fumantes com infarto agudo do miocárdio. Arquivos Catarinenses de Medicina, v. 36, n. 2, p. 34-40, 2007.

SILVEIRA, E.L. et al. Prevalência e distribuição de fatores de risco cardiovascular em portadores de doença arterial coronariana no Norte do Brasil. Revista da Faculdade de Ciências Médicas de Sorocaba, v.20, n.3, p.167-173, 2018.

SIQUEIRA, D.G.B. et al. Diferenças entre sexos nos determinantes da obesidade abdominal em adultos de 40 anos ou mais: estudo de base populacional. Rev. Nutr. [online], v. 28, n. 5, p. 485-496, 2015.

TEIXEIRA, C.S.; SANCHES, S.B.; VIVAS, I.S. Prevalência de fatores de risco em pacientes pós-infarto agudo do miocárdio. Unisanta Health Science, v.1, n.1, p.1-18, 2017.

TUAN, T.S.; VENÂNCIO, T.S.; Nascimento L.F.C. Efeitos da Exposição a Poluentes do Ar no Infarto Agudo do Miocárdio, Segundo Gêneros. Arquivos Brasileiros de Cardiologia, v.107, n.3, p.216-222, 2016. 